\title{
USO DE MASCARILLAS EN TIEMPOS DE COVID-19: ALGUNAS MANIFESTACIONES EN LA PIEL DEL PERSONAL DE LA SALUD
}

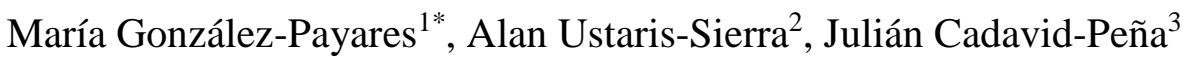 \\ ${ }^{1,2}$ Universidad del Norte, Barranquilla, Colombia \\ ${ }^{3}$ Universidad CES, Medellín, Colombia \\ *Autor de correspondencia: mdpayares@uninorte.edu.co
}

Recibido Noviembre 2020; Aceptado Diciembre 2020

\begin{abstract}
Resumen - Gracias al rápido desarrollo de la pandemia por COVID-19, muchos profesionales de la salud han presentado lesiones de piel en sus rostros debido al uso prolongado de equipos de protección personal, hasta la fecha se han descrito cinco formas o presentaciones clínicas de lesiones faciales: pseudo-sabañones, brotes vesiculares, lesiones urticariales, brote maculopapular y necrosis o livedo. Se realizó una revisión bibliográfica no sistemática basado en la búsqueda de artículos utilizando las palabras de filtro "Dermatología, Piel, EPP, mascarillas y COVID19", en las bases de datos electrónicas Redalyc, Dialnet, Google Académico y Scielo. Las lesiones por presión relacionadas con dispositivos médicos son producidas como resultado de una presión de mayor intensidad en áreas pequeñas, o una presión de menor intensidad, pero de larga duración, en áreas más grandes, en combinación con fricción y cizallamiento. Para lo que se han planteado una serie de medidas que se clasifican en básicas y específicas.
\end{abstract}

Palabras clave: COVID-19, Equipo de Protección Personal, Dermatología, Mascarilla, Personal de la salud.

\section{USE OF MASKS IN TIMES OF COVID-19: SOME MANIFESTATIONS ON THE SKIN OF HEALTHCARE PERSONNEL}

\begin{abstract}
Due to the rapid development of the COVID-19 pandemic, many health professionals have presented skin lesions on their faces, due to the prolonged use of personal protective equipment, to date five forms or clinical presentations have been described: pseudo-chillblain, other vesicular outbreaks, urticarial lesions, maculopapular outbreak and necrosis or livedo. A non-systematic bibliographic review based on a search for articles was carried out using the filter words "Dermatology, Skin, PPE, masks and COVID-19", in the electronic databases Redalyc, Dialnet, Google Academic and Scielo. Pressure injuries related to medical devices are produced as a result of a pressure of greater intensity in small areas, or a pressure of less intensity, but of long duration, in larger areas, in combination with friction and shear. For which a series of of measures that are classified as basic and specific.
\end{abstract}




\section{Uso de mascarillas en tiempos de COVID-19: Algunas manifestaciones en la piel del personal de la salud}

Keywords: COVID-19, Personal Protective Equipment, Dermatology, Mask, Health personnel.

\section{Introducción}

El virus SARS-CoV-2 es muy contagioso y se transmite rápidamente de persona a persona a través de la tos o secreciones respiratorias, y por contactos cercanos; las gotas respiratorias de más de cinco micras son capaces de transmitirse a una distancia de hasta dos metros, y las manos o los fómites contaminados con estás secreciones seguido del contacto con la mucosa de la boca, nariz u ojos (Fong et al., 2020; Maguiña et al., 2020)., se ha hecho necesario la utilización con mayor frecuencia de diferentes equipos de protección personal (EPP) en los profesionales de la salud implicados en la atención a pacientes infectados o sospechosos, lo que ha conllevado a la aparición de lesiones cutáneas, antes estas eran poco prevalentes y se conoce muy poco acerca de su alcance, prevención y tratamiento (Garcés et al., 2020).

En tal sentido, la opción del uso prolongado de EPP suele darse porque implica una menor autoinoculación, por ende, un riesgo más bajo de transmisión del virus. Pero esto también puede generar lesiones en la piel, en este contexto, cabe resaltar que el desarrollo de cualquier tipo de lesión cutánea del profesional de la salud aumenta su vulnerabilidad al contagio, y facilita la entrada de la enfermedad. Esto lo alejaría de sus actividades laborales en un momento en que su papel es de vital importancia (da Silva et al., 2020).

Hay poca evidencia sobre el alcance epidemiológico de las lesiones cutáneas mencionadas, solo un trabajo centrado en profesionales de la salud de China publicado por Zhang et al. (2020); es casi que obligatorio el conocer la epidemiología, las características y los factores relacionados con la utilización de los diferentes tipos de EPP y de la prevención y el tratamiento de dichas lesiones, puede ser de gran utilidad para conocer el alcance del problema y definir estrategias para su prevención y tratamiento en posibles pandemias futuras (González, 2020).

La Organización Mundial de la Salud señala la utilización permanente de Mascarilla, sin embargo, estas recomendaciones plantean desafíos serios e impactantes, descritos en informes de profesionales chinos e italianos, que deben ser reconocidos, analizados y comprendidos para poder desarrollar estrategias adecuadas de afrontamiento (Santos et al., 2020). Durante los comienzos de la pandemia, fue posible observar que la piel es más vulnerable a la agresión química y física, ya que la frecuencia del uso de productos y equipos es mayor. El aumento de la demanda, a su vez, dio lugar a una escasez mundial de los mismos y, por consiguiente, los profesionales de la salud que trabajan en diferentes escenarios de la atención sanitaria no siempre disponen de estos materiales en cantidades suficientes (Feng et al., 2020). En este sentido, una nota técnica recomienda que las mascarillas podrían, excepcionalmente, usarse durante un período y/o número de veces mayor que el recomendado (Lai et al., 2020).

En palabras de Carrascosa et al. (2020), la rápida propagación de la infección por el coronavirus SARS-CoV-2 y la enfermedad asociada (COVID-19) representan un grave problema de salud pública. La pandemia ha condicionado la suspensión de la actividad de la mayor parte de Clínicas de dermatitis de contacto y la práctica de pruebas epicutáneas debido a la adaptación de los centros hospitalarios a la prioridad del tratamiento de esta infección. 


\section{Uso de mascarillas en tiempos de COVID-19: Algunas manifestaciones en la piel del personal de la salud}

El riesgo de propagación del SARS-CoV-2 en las unidades de salud es desconocido, y si bien las estancias comunes de dichas unidades siguen los protocolos de desinfección similares al resto de servicios hospitalarios, en las unidades de dermatitis de contacto, organizadas habitualmente en forma de gabinetes con participación activa de facultativos y enfermería, tiene lugar un contacto prolongado en el tiempo, con varias visitas en pocos días y muy cercano con respecto al paciente que condiciona problemas específicos a la hora de la seguridad de propagación del SARS-CoV-2 entre ellos, y entre los pacientes y el personal sanitario (Moreno, 2020). Por todo lo hasta aquí expuesto, el objetivo de este trabajo es hacer una revisión de como las mascarillas han traído como consecuencia manifestaciones en la piel del personal de la salud en tiempos de COVID-19, con miras al desarrollo de estrategias y alternativas para una mayor ergonomía en el ejercicio de su trabajo.

\section{Materiales y Métodos}

Se desarrolló una revisión bibliográfica desde la declaración de pandemia al mes de octubre Se hicieron búsquedas en las bases de datos electrónicas Redalyc, Dialnet, Science Direct, Google Académico y Scielo. Los términos empleados para la búsqueda fueron: Lesiones cutáneas, adhesivos tisulares, dispositivos médicos y úlceras por presión; combinados con COVID-19 y Equipos de Protección Personal.

\section{Resultados y Discusión}

El brote pandémico de COVID-19 planteó un nuevo tema de preocupación mundial desde diciembre del 2019: las lesiones cutáneas en el personal de salud usuaria de EPP, con el subsecuente deterioro del estado de salud y de la calidad de vida; se ha encontrado que el uso continuo y prolongado el mismo se asocia a una mayor prevalencia de estas, manifestándose en mayor frecuencia como xerosis, descamación, eritema y maceración en múltiples localizaciones anatómicas, siendo las manos, el puente nasal y las mejillas las regiones más afectadas (ErizeHerrera et al., 2020).

La piel es uno de los órganos más afectados por COVID-19 y a la fecha se han descrito cinco formas o presentaciones clínicas: Pseudo-sabañones (áreas acrales eritematosas con vesículas y pústulas), otros brotes vesiculares, lesiones urticariales, brote maculopapular y necrosis o livedo. También se han visto múltiples manifestaciones cutáneas secundarias al uso de los equipos de protección personal, tales como mascarillas, gafas, gorros, y guantes, especialmente en el personal de la salud. En esta revisión se describen las manifestaciones asociadas al uso de tales equipos y se recopilan las recomendaciones publicadas hasta ahora para prevenirlas y tratarlas (Piedrahita et al., 2020).

La Organización Mundial de la Salud (OMS) recomienda el uso de los EPP para los profesionales sanitarios, que mantienen una atención directa con el paciente, el uso de mascarillas es sumamente necesario, además como ente de control sanitario, reconoce la necesidad imperante de utilizar mascarillas, barbijos o tapabocas para protección respiratoria, pero establece la recomendación de que estos no sean utilizados durante más de 4 o 6 horas seguidas, debido a la incomodidad causada por los mismos (Saavedra, 2020). 


\section{Uso de mascarillas en tiempos de COVID-19: Algunas manifestaciones en la piel del \\ personal de la salud}

En condiciones normales en América Latina, los profesionales sanitarios laboran alrededor de 8 horas diarias, las condiciones sanitarias dadas por la pandemia llevaron a tomar decisiones administrativas a nivel político de establecer nuevos horarios, así es como en muchos países se pautaron jornadas laborales de 12 y 24 horas. Esto con el objetivo de optimizar el recurso humano (entre otros factores dado el alto número de contagios entre los profesionales de la salud) y ahorrar considerablemente el gasto de EPP (debido a la escasez y dificultad de adquirirlos). El uso prolongado de tapabocas, especialmente N-95 / FFP2 / mascarillas doble filtro y gafas de protección, puede aumentar el riesgo de lesiones por presión y/o fricción en el rostro (Delgado et al., 2020).

En el estudio de encuesta descriptiva de Qin et al. (2020) en un hospital estatal de China, durante la pandemia de COVID-19 $(n=61)$, se informó que de los trabajadores sanitarios que utilizan mascarilla N95, el 68,9\% experimentó lesiones en el puente nasal y el 27,9\% prurito en la cara. Recientemente se reportó en China una prevalencia del $97 \%$ de lesiones en la piel del personal de salud que está en primera línea.

Algunas de las mascarillas N95 contienen formaldehído por lo que su uso se ha asociado a dermatitis de contacto facial, lo cual se ha evidenciado en brotes de síndrome respiratorio agudo grave. Además, se han descrito tres factores importantes para evitar las injurias por mascarilla N95, las cuales son: intensidad de la presión, la duración de la presión y la tolerancia individual. Se ha demostrado que las máscaras quirúrgicas contienen formaldehido, metildibromo glutaronitrilo y otros conservantes, que son potentes desencadenantes de dermatitis de contacto (Garcés-Abad et al., 2020).

Otras dermatosis asociadas al uso prolongado de máscaras y gafas incluyen lesiones por presión, urticaria, dermatitis de contacto, xerosis y empeoramiento de dermatosis preexistentes. Según un informe de Singapur durante el brote de SARS en 2003, el 35,5\% de los trabajadores sanitarios informaron manifestaciones dermatológicas por el uso de mascarillas N95, así: acné $(59,6 \%)$, prurito facial $(51,4 \%)$ y erupción cutánea $(35,8 \%)$. (Kantor, 2020).

Hasta el momento no existen guías o ensayos clínicos que valoren el manejo o prevención de las lesiones por el uso de equipo de protección personal, por lo que en China se realizó un consenso de expertos para emitir recomendaciones para protección de la piel y mucosas en trabajadores de la salud expuestos a pacientes con COVID-19 (Mendoza et al., 2020).

En México, de acuerdo con Pérez \& Arellano (2020), se inició la reconversión del Hospital General de México para la atención de pacientes en áreas de urgencias, torres quirúrgicas, unidades de hospitalización, así como la capacitación de personal de salud: médicos de base, residentes, enfermería, personal administrativo, personal de intendencia y camilleros para la correcta atención y movilización de pacientes dentro del hospital. Esto conlleva, por tanto, a que se utilice por tiempo prolongado el equipo de protección personal, por lo que nuestro servicio, con apoyo de nuestras autoridades, se tomó la tarea de abrir una unidad especializada para la atención de estas complicaciones. Con esta acción, se suma el dermatólogo en esta pandemia, no sólo con la descripción y clasificación de las dermatosis asociadas propiamente 


\section{Uso de mascarillas en tiempos de COVID-19: Algunas manifestaciones en la piel del personal de la salud}

con la infección, sino en el área de la salud ocupacional, en la atención de nuestros compañeros que se encuentran en la primera línea atendiendo directamente a los pacientes.

En términos generales, por muy útil que sea, el uso de la mascarilla puede provocar escoriaciones en la piel del puente de la nariz, la barbilla, las mejillas e incluso detrás de las orejas. Algunas personas desarrollan prurito o eritema. Las condiciones de sudor o humedad bajo la mascarilla pueden provocar brotes de acné y otros problemas de la piel. Para aliviar estas complicaciones del uso de la mascarilla durante la pandemia de COVID-19, se pueden seguir los lineamientos descritos por Aranaz et al. (2020):

- Adopta una rutina regular de cuidado de la piel. Limpia e hidrata tu cara antes y después de usar la mascarilla. Elige productos etiquetados como no comedogénicos. Pero no uses productos con petrolato. El petrolato es un ingrediente común en cremas y ungüentos "de uso intensivo", como la vaselina. Puede interferir en la función de la mascarilla, especialmente las mascarillas N95 utilizadas por algunos trabajadores de atención médica. Si tu piel tiende a ser grasosa, usa una crema hidratante soluble en agua.

- Usa un ungüento o un vendaje de barrera. Para prevenir o aliviar las lesiones de la piel causadas por la presión o fricción de las mascarillas, una fina capa de óxido de zinc puede calmar y proteger la piel. El óxido de zinc es un protector de la piel que se utiliza a menudo para la dermatitis del área del pañal o la piel muy agrietada. Úsalo en el puente de la nariz o detrás de las orejas. También puedes intentar colocar una venda entre los puntos de presión de la piel y la mascarilla. Existen algunos vendajes o apósitos que proporcionan un alivio refrescante y se ajustan a la piel, permitiendo al mismo tiempo el uso apropiado de la mascarilla.

- Alivia las ampollas. Si se forma una ampolla, mantenla limpia y aplica un ungüento antibiótico. Usa una venda para crear una barrera protectora entre la ampolla y la mascarilla.

- Trata el acné. Si desarrollas acné, limpia tu piel regularmente. Usa un humectante soluble en agua. Busca productos para el tratamiento del acné que contengan peróxido de benzoílo o ácido salicílico. Además, evita reventar u oprimir los granos, que pueden contribuir a la inflamación e infección de la zona.

Como medidas preventivas se recomienda usar hidratantes antes de ponerse la mascarilla; sin embargo, existe controversia sobre su uso: la NPIAP (siglas en inglés de National Pressure Injury Advisory Panel) no lo recomienda, ya que no hay evidencia científica suficiente que avale su uso; además, se cuestiona si esto puede aumentar el riesgo de adquirir la infección por SARS-CoV-2. Sugieren disminuir la duración de la presión retirando la mascarilla durante 15 minutos cada dos horas en lugares donde no haya contacto con pacientes o si esto no es posible, por lo menos durante cinco minutos cada dos horas. Para las líneas de presión al retirarse los elementos de protección se recomienda la aplicación de compresas frías por 20 minutos cada dos a tres horas y luego hidratantes, evitando el calor y agentes posiblemente irritantes (Atzori et al., 2020). 


\section{Uso de mascarillas en tiempos de COVID-19: Algunas manifestaciones en la piel del personal de la salud}

A pesar de esto, Figueroa (2020) detalla que la adherencia a las medidas de protección es fundamental, ya que no seguir las recomendaciones se ha asociado a infecciones en el personal de la salud. Para disminuir los riesgos de manipulación de la cara secundaria a la irritación, es importante explicarle previamente al personal que puede aparecer irritación en los puntos de roce o contacto. En caso de usarse algún producto tópico para mejorar o prevenir estos síntomas, se debe aplicar con extremo cuidado, por ejemplo, usando un aplicador estéril o previo al ingreso de salud.

\section{Conclusiones}

De los resultados mostrados, de su análisis y de su discusión, se pueden obtener las siguientes conclusiones, sobre la utilización de mascarillas en tiempos de COVID-19 y algunas manifestaciones en la piel del personal de la salud: El uso prolongado de EPP tipo mascarilla se asocia a manifestaciones cutáneas principalmente exacerbación de cuadros de acné o de dermatosis previas así como lesiones producto del contacto con los componentes del EPP. Existen recomendaciones importantes para los trabajadores de la salud para evitar la aparición y/o minimizar el impacto de dichas lesiones. En el momento el comportamiento de la pandemia a futuro es desconocido por lo que es muy probable que el uso de mascarillas se prolongue por un largo periodo de tiempo, de ahí la importancia de conocer y seguir las recomendaciones ya mencionadas.

\section{Referencias}

Aranaz, J. M. A., de Castro, M. T. G. V., Vicente-Guijarro, J., Peribáñez, J. B., Haro, M. G., Valencia-Martín, J. L., ... \& Martín, J. L. V. (2020). Mascarillas como equipo de protección individual durante la pandemia de COVID-19: cómo, cuándo y cuáles deben utilizarse. Journal of Healthcare Quality Research, 35(4), $245-252$.

Atzori, L., Ferreli, C., Atzori, M. G., \& Rongioletti, F. (2020). COVID-19 and impact of personal protective equipment use: From occupational to generalized skin care need. Dermatologic therapy, 33(4), e13598. https://doi.org/10.1111/dth.13598

Carrascosa, J. M., Morillas, V., Bielsa, I., \& Munera-Campos, M. (2020). Manifestaciones cutáneas en el contexto de la infección por SARS-CoV-2 (COVID-19). Actas Dermo-Sifiliográficas.

da Silva Brandão, E., Mandelbaum, M. H. S. A., Faria da Silva, L., Reis, B. L., \& Tonole, R. (2020). Prevención de lesiones cutáneas causadas por productos y equipos de protección personal durante COVID-19: revisión de alcance. Journal of wound care, 29(LatAm sup 3), 6-12.

Delgado, M. T. B., Quijije, W. F. D., Flores, B. I. P., \& Velázquez, M. D. R. H. (2020). estrés laboral en el personal de enfermería del área de uci durante la pandemia de covid 19. unesum-Ciencias. Revista Científica Multidisciplinaria. ISSN 2602-8166, 4(3), 41-50.

Erize-Herrera, J. A., García-Mireles, V., Uh-Sánchez, I., Felix-Téllez, F., Encarnación-Martínez, M., \& EstradaAguilar, L. (2020). Manifestaciones dermatológicas en profesionales de salud asociadas al uso de equipo de protección personal para la atención de los pacientes con infección por COVID-19 en los hospitales del área metropolitana de la ciudad de México. Piel.

Feng, S., Shen, C., Xia, N., Song, W., Fan, M., \& Cowling, B. J. (2020). Rational use of face masks in the COVID19 pandemic. The Lancet Respiratory Medicine. 


\section{Uso de mascarillas en tiempos de COVID-19: Algunas manifestaciones en la piel del personal de la salud}

Figueroa, L. (2020). Estrategias para la prevención y control de las infecciones asociadas a la atención sanitaria. Revista del Hospital" Dr. Emilio Ferreyra", 1(2), e35-e44.

Fong-Silva, W., Severiche-Sierra, C., \& Fong-Amarís, W. (2020). Sistemas de acondicionamiento de aire y ventilación como foco infeccioso de SARS CoV-2 en hospitales, clínicas e instituciones de salud. IPSA Scientia, Revista científica Multidisciplinaria, 5(1), 28-35. https://doi.org/10.25214/27114406.960

Garcés-Abad MA, Galeano-Piedrahita E, Uribe-Zapata DE, Gaviria-López AF, Pérez-Madrid C. Compromiso cutáneo secundario al uso del equipo de protección contra COVID-19 en el personal de la salud. Rev CES Med. 2020; Especial COVID-19: 8-13.

González-Payares, M. (2020). COVID-19 en Iberoamérica: Un acercamiento desde la dermatología. IPSA Scientia, Revista científica Multidisciplinaria, 5(1), 79-87. https://doi.org/10.25214/27114406.1022

Kantor, J. (2020). Behavioral considerations and impact on personal protective equipment use: Early lessons from the coronavirus (COVID-19) pandemic. Journal of the American Academy of Dermatology, 82(5), 1087-1088.

Lai J, Ma S, Wang Y, Cai Z, Hu J, Wei N et al. Factors Associated With Mental Health Outcomes Among Health Care Workers Exposed to Coronavirus Disease 2019. 2020. [citado em 1 abril 2020]. Disponível em: https://doi:10.1001/jamanetworkopen.2020.3976.

Maguiña Vargas, C., Gastelo Acosta, R., \& Tequen Bernilla, A. (2020). El nuevo Coronavirus y la pandemia del Covid-19. Revista Medica Herediana, 31(2), 125-131.

Mendoza, J. L. A., Beltrán, N., Estrada, V. H. N., Bolaños, E. R., Gómez, C. P., Rebolledo, C. E., ... \& Camargo, R. (2020). Declaración de consenso en medicina crítica para la atención multidisciplinaria del paciente con sospecha o confirmación diagnóstica de covid-19. Acta Colombiana de Cuidado Intensivo, 20(4), 287-333.

Moreno-Martínez, F. L., Moreno-López, F. L., \& Oroz Moreno, R. (2020). Repercusión cardiovascular de la infección por el nuevo coronavirus SARS-CoV-2 (COVID-19). CorSalud, 12(1), 3-17.

Pérez-García, U., \& Arellano-Mendoza, M. I. (2020). Labor de Dermatología en la atención a reacciones cutáneas adversas por uso de equipo de protección personal durante la pandemia por COVID-19 Dermatology work in attention to adverse skin reactions due to the use of personal protective equipment during the COVID-19 pandemic. Dermatología Revista Mexicana, 64(4), 487-490.

Piedrahita, E. G., Abad, M. A. G., Zapata, D. E. U., Gaviria, A. F., \& Madrid, C. P. (2020). Compromiso cutáneo secundario al uso del equipo de protección contra COVID-19 en el personal de la salud. CES Medicina, 34, 8-13.

Qin, C., Zhou, L., Hu, Z., Zhang, S., Sheng, Y., Tao, Y., Xie, C., Shang, K., Wang, W. y Tian, D. (2020). Dysregulation of immune response in patients with COVID-19 in Wuhan, China. Clinical Infectious Diseases. doi:10.1093/cid/ciaa248

Saavedra Trujillo, C. H. (2020). Consenso colombiano de atención, diagnóstico y manejo de la infección por SARS-COV-2/COVID-19 en establecimientos de atención de la salud. Recomendaciones basadas en consenso de expertos e informadas en la evidencia. Infectio, 24(3), 186-261.

Santos-López, M., Jaque-Ulloa, D., \& Serrano-Aliste, S. (2020). Métodos de Desinfección y Reutilización de Mascarillas con Filtro Respirador Durante la Pandemia de SARS-CoV-2. International journal of odontostomatology, 14(3), 310-315.

Zhang Y, Cao W, Xiao M, Li Y, Yang Y, Zhao J, Zhou X, Jiang W, Zhao YQ, Zhang SY, Li TS. [Clinical and coagulation characteristics of 7 patients with cri-tical COVID-2019 pneumonia and acroischemia]. Zhonghua Xue Ye Xue Za Zhi. 2020;41(0):E006. doi: 10.3760/cma.j.issn.0253-2727.2020.0006 